\title{
Erfolg durch Leistung?
}

\section{Ein Forschungsüberblick zum Thema Wissenschaftskarrieren}

\author{
Von Christiane Gross und Monika Jungbauer-Gans
}

Zusammenfassung: Der Beitrag präsentiert den Forschungsstand zu den Bestimmungsfaktoren erfolgreich verlaufender wissenschaftlicher Karrieren. Von Interesse ist dabei insbesondere, ob und inwiefern nicht-meritokratische Kriterien zum Erklimmen der wissenschaftlichen Karriereleiter förderlich sind und durch welche Mechanismen diese Effekte zustande kommen. Zur Darstellung eines weitgehend als legitim empfundenen, leistungsorientierten Wissenschaftsverständnisses wird das wissenschaftliche Ethos nach Robert K. Merton präsentiert und anschließend mit den Befunden bisheriger Studien konfrontiert. Die Sekundäranalyse verweist auf die enorme Bedeutung sozialer Netzwerke, welche in vielfacher Hinsicht (u.a. über das Renommee der Institutionen) mit wissenschaftlicher Produktivität konfundiert sind. Zudem scheint zumindest der direkte Effekt askriptiver Merkmale wie Geschlecht und ethnischer Zugehörigkeit auf wissenschaftlichen Erfolg an Stärke eingebüßt zu haben, wenngleich indirekte Mechanismen noch weiterhin wirksam sind.

\section{Einleitung}

Das Ziel der Wissenschaft ist es, innovative Erkenntnisse zu erarbeiten und zum Wissensund Wissenschaftsfortschritt beizutragen. Wissenschaftliches Wissen erhebt den Anspruch, >wahres< bzw. >gesichertes< Wissen zu sein (Weingart 2003, S.7). An diesem epistemologischen Kern der modernen Wissenschaft haben auch wissenssoziologische (konstruktivistische) Ansätze wenig ändern können. Sie stellen die Erklärung des Inhalts der Forschungsergebnisse gegenüber ihrer äußeren Gestalt (Wachstum, Innovativität und Themenwahl) in den Vordergrund (Schimank 1995) und haben auf die Bedeutung sozialer Prozesse und kultureller Faktoren bei der Genese und Anerkennung von wissenschaftlichem Wissen hingewiesen. Obwohl soziale Prozesse, aber auch Unvermögen zu Umwegen und Irrtümern führen können, stellt die >wahre< bzw. >gesicherte< Erkenntnis letztlich das Ziel wissenschaftlichen Schaffens dar.

In diesem Beitrag geht es nicht in erster Linie um die Mechanismen der gesellschaftlichen Wissensproduktion, sondern um die Mechanismen, nach denen wissenschaftliche Karrieren in diesem gesellschaftlichen Kontext funktionieren. Welche Kriterien beeinflussen den Verlauf von Karrieren in der Wissenschaft? An welchen Kriterien orientieren sich die Akteure (Personen, die eine wissenschaftliche Karriere anstreben bzw. Personen, die Auswahlentscheidungen treffen)? Ein unausgesprochener Konsens besteht darin, dass Kriterien, die letztendlich dem Ziel einer effektiven Wissensproduktion und dem wissenschaftlichen Fortschritt dienen, ausschließlich die Leistung der Personen berücksichtigen sollten. Dabei stellt sich allerdings die Frage, ob neben diesen auch nicht-meritokratische bzw. askriptive Merkmale wie das Geschlecht oder die soziale und ethnische Herkunft die individuellen Karrierechancen beeinflussen. Doch bevor auf die Darstellung von Kriterien und den diesbezüglichen Forschungsstand eingegangen wird, wird das von Robert K. Merton beschriebene Ethos der Wissenschaft als Grundlage der Argumentation eingeführt. Die Ausführungen zeigen, dass wissenschaftliche Normen die wesentlichen Institutionen sind, die einerseits die Verpflichtung der Wissenschaftler auf den gesellschaftlichen Fortschritt durch geeignete Anreize sicherstellen, andererseits dennoch Freiräume für Kreativität bieten.

Die Akteure werden dabei nicht als Marionetten institutioneller Regelungen gesehen, sondern als eigeninteressierte Akteure, die an substanziellen Fragestellungen, am Erwerb von 
sozialem Kapital (Reputation) und an forschungsdienlichen Ressourcen, Autonomie und geringer Konkurrenz orientiert sind (Schimank 1995). Vorfindbare Normen orientieren das Eigeninteresse, das strategische Handlungspotenzial und legen die legitimen Mittel und Wege der Zielerreichung fest.

\section{Wissenschaftliche Normen und die Rekrutierung von Nachwuchs}

Normen und Werte wissenschaftlichen Handelns wurden bereits bei Max Weber (1968) thematisiert. Weber beschrieb, wie allgemeine gesellschaftliche Normen die Auswahl von Forschungsfragen und wissenschaftliche Relevanzen begründen können, Werte als Gegenstand von Forschungsfragen dienen und eine allgemeine Basis wissenschaftlichen Handelns darstellen. Mit »Werturteilsfreiheit « meint Weber die Ausklammerung von Werten aus wissenschaftlichen Aussagen. Werte dürfen nicht die inhaltlichen Schlussfolgerungen wissenschaftlicher Aussagen beeinflussen. Welche konkreten Werte und Normen wissenschaftliches Handeln steuern, wurde von Robert K. Merton (1985[1942]) beschrieben. Er beschäftigte sich mit den sozialen Normen und Regeln, die die Produktion und Verbreitung > wahren< Wissens sicherstellen sollen. Diese Normen und Regeln bezeichnet er als »Ethos der Wissenschaft«. Er unterscheidet vier Komplexe: (1) Universalismus, (2) Kommunismus, (3) Uneigennützigkeit und (4) organisierten Skeptizismus.

(1) Der Universalismus der Wissenschaft führt zur Forderung, dass »Wahrheitsansprüche [...] vorab aufgestellten, unpersönlichen Regeln unterworfen werden müssen: sie müssen mit der Beobachtung und dem bisher bestätigten Wissen übereinstimmen.« (Merton 1985, S.90). Der Universalismus hat zur Folge, »dass dem Talent die Chance einer wissenschaftlichen Karriere nicht verwehrt werden darf. Wissenschaftliche Karrieren aus anderen als Gründen des Talents zu behindern, beeinträchtigt die Erweiterung von Wissen und Erkenntnis« (Merton 1985, S.91f.).

(2) Kommunismus, im Sinn des gemeinschaftlichen Besitzes von Gütern, bewirkt, dass die substanziellen Erkenntnisse der Wissenschaft ein Produkt gesellschaftlicher Zusammenarbeit sind und der Gemeinschaft gehören (Merton 1985, S.93). Dem Forscher gebührt lediglich Anerkennung und Ansehen, aber nicht die alleinige Verfügung über die Erkenntnisse. Diese Norm impliziert die Forderung nach Veröffentlichung der Forschungsergebnisse. Daher sind Patente und Geheimhaltung nicht mit dieser Norm vereinbar. Aus der Norm des Kommunismus lässt sich eine hohe Wertschätzung von Publikationen, insbesondere die Betonung von Originalität bei der Bewertung individueller Leistungen, in und für die Forschung herleiten.

(3) Weiterhin ist die Uneigennützigkeit für die Wissenschaft konstitutiv. Merton (1985) setzt Uneigennützigkeit nicht mit Altruismus gleich. Vielmehr ist das Verhalten der Wissenschaftler geprägt durch ein System institutioneller Kontrolle, das uneigennütziges Handeln belohnt. Wichtig ist hierbei die von Fachkollegen ausgeübte Kontrolle, die den Missbrauch von Fachautorität und die Schaffung von Pseudowissenschaften verhindern kann (Merton 1985, S.98). Für den einzelnen Wissenschaftler bedeutet dieses Prinzip, dass sich seine Arbeiten in der Begutachtung von Fachkollegen bewähren müssen. Bei der Bewertung wissenschaftlicher Produktivität nehmen deshalb Arbeiten, die einen peer-review Prozess durchlaufen haben, eine besondere Rolle ein.

(4) Der organisierte Skeptizismus stellt ein methodologisches und institutionelles Gebot dar. Die unvoreingenommene Prüfung von Glaubensüberzeugungen mit empirischen und logischen Maßstäben bringt die Wissenschaft immer wieder in Konflikte mit anderen Institutionen, die diese Glaubensüberzeugungen vertreten (Merton 1985, S.99). In methodologischer Hinsicht gebietet diese Norm die Formulierung falsifizierbarer Forschungsfragen und deren systematische Prüfung. Für den einzelnen Forscher heißt dies, dass Forschungen sorgfältig 
und nach dem Stand des Wissens durchgeführt werden müssen, und insbesondere Beschönigungen der Ergebnisse oder gar Fälschungen unterbleiben müssen. Abgesehen von offensichtlich gewordenen Missbräuchen ist diese Norm für Individuen nur mit einer differenzierten Analyse der vorgelegten Arbeiten zu überprüfen.

Insbesondere die vierte Norm rückt die Position Mertons in die Nähe der wissenschaftstheoretischen Position des Kritischen Rationalismus. Es stellt sich die Frage, ob der Merton'sche Ethos in wissenschaftlichen Paradigmen, die dieser Position explizit widersprechen, Akzeptanz finden könnte (vgl. dazu Weingart 2003, S.17). Ein weiterer Kritikpunkt lässt sich aus den Ansätzen von Kuhn und Feyerabend ableiten, die gezeigt haben, dass das Verhalten von Wissenschaftlern nicht durch allgemein gültige Normen, sondern durch spezifische Paradigmen gesteuert und dies nach Feyerabend wenig zielführend sei (Feyerabend 1986[1976]; Kuhn 1973[1962]). Dennoch stellen herrschende Paradigmen kein überzeugendes Gegenargument dar unter den beiden Annahmen, dass man Erstens innerhalb der einzelnen Paradigmen gut beraten ist Personen auszuwählen, die den von Merton beschriebenen Normen genügen, und Zweitens diese ausgewählten Personen dadurch leistungsfähiger im Sinne des wissenschaftlichen Fortschritts sind. Darüber hinaus gibt es einen Wettbewerb zwischen den Paradigmen, der sich letztlich auch von der Leistungsfähigkeit des Paradigmas selbst und seiner Protagonisten nährt. Ein zweiter Kritikpunkt richtet sich gegen die Interpretation des Ethos der Wissenschaft als empirische Beschreibung des Verhaltens von Wissenschaftlern. Zwar kann man Indizien dafür finden, dass Mertons Ethos tatsächlich eine analytische Verdichtung von Verhaltensregeln darstellt (Weingart 2003, S.18), aber man sollte Normen und Verhalten nicht gleichsetzen. Tatsächliches Verhalten, Anreize zu Fehlverhalten und Widersprüche zwischen den einzelnen Normen sind nicht grundsätzlich als Widerlegung der Normen zu betrachten. Vielmehr haben Fälle von wissenschaftlichem Betrug zu einer Kodifizierung des wissenschaftlichen Ethos geführt, die den Formulierungen Mertons relativ nahe kommen (Weingart 2003, S.21). Merton gebührt damit das Verdienst, die zentrale Frage >Wie ist gesichertes Wissen möglich?< gestellt zu haben. Er stellt aber nicht nur diese Frage, sondern liefert auch eine soziologische Antwort darauf, indem er aufzeigt, über welche Institutionen und Mechanismen Wissenschaft versucht, diesem Ziel gerecht zu werden. In diesem Beitrag geht es darum, die bereits präsentierten normativen Forderungen an Funktionsweisen und Selektionskriterien universitärer Praxis mit der empirischen Evidenz bzw. dem Forschungsstand zu der Thematik abzugleichen.

Nimmt man die Implikationen des wissenschaftlichen Ethos zusammen, führt dies zu der Folgerung, dass eine unpersönliche Leistungsbewertung konstitutiv für eine Wissenschaft im Merton'schen Sinne ist. Selektionsmechanismen bei der Auswahl von Forscherinnen und Forschern sollten zur Auswahl leistungsfähiger Personen führen, damit diese Personen einen hohen Beitrag zum wissenschaftlichen Fortschritt leisten können. In der Praxis verwendet man in der Regel Indikatoren bisheriger Leistungen als Proxies zur Einschätzung künftiger Leistungsfähigkeit.

\section{Auswahlprozesse}

Bevor auf die Bedeutung konkreter Kriterien wie wissenschaftlicher Produktivität oder Drittmitteleinwerbung eingegangen wird, werden die grundlegenden Mechanismen des Auswahlprozesses erläutert. Wir beschränken uns hierbei auf Berufungsverfahren von Professoren und lassen Selektionsmechanismen auf den vorherigen Karrierestufen außer Acht in der Annahme, dass der grundlegende Tenor der Personalauswahl sich hier ebenfalls widerspiegeln sollte.

Die Beschäftigung von Hochschulprofessoren/innen erfolgt in Deutschland in der Regel als Beamte auf Lebenszeit. Einige Bundesländer sind mittlerweile dazu übergegangen, Erst- 
rufe nur noch auf Zeit zu vergeben, um nach einem bestimmten Zeitraum eine Evaluation durchzuführen und die Beschäftigung zu entfristen. ${ }^{1}$ Die akademischen Positionen waren bei Berufungen bis einschließlich 2004 gegliedert in C4-, C3- sowie C2-Professuren, die sich in Bezahlung und Ausstattung unterschieden. Mit der Reform des Besoldungsrechts 2005 wurden diese Positionen abgelöst durch W3- und W2-Professuren, die sich auszeichnen durch ein konstantes, d.h. nicht mehr mit dem Lebensalter steigendes, Grundgehalt und einem erheblichen Anteil an Leistungsbezügen in Relation zum Grundgehalt. Darüber hinaus wurde im Jahr 2002 die Juniorprofessur (entlohnt nach W1) eingeführt. Juniorprofessuren sind befristete Stellen von zwei mal drei Jahren, die aber teilweise mit einem so genannten tenure track ausgestattet sind, der eine Berufung auf eine W2-Professur nach Ablauf der Juniorprofessorenstelle ermöglicht.

Berufungskommissionen werden mit professoraler Mehrheit von den Fakultäten unter Vorsitz des Dekans eingesetzt. Die Stellen werden national oder international öffentlich ausgeschrieben. Nach einer Vorauswahl in der Berufungskommission bewerten mehrere externe Gutachter die in die engere Wahl gezogenen Kandidatinnen und Kandidaten. Berufungslisten werden von der Berufungskommission vorgeschlagen und vom Fakultätsrat sowie dem Senat der Universität verabschiedet. Berufungen schließlich spricht meist das Ministerium bzw. die Universitätsleitung aus. Die Bezüge und die Ausstattung des Lehrstuhls werden mit der Universitätsleitung und den Fakultäten ausgehandelt.

Kriterien für die Auswahl von Kandidaten sind Forschungs-, Lehr- und Verwaltungstätigkeiten, wobei in der Regel - nicht zuletzt wegen der guten Dokumentierbarkeit durch Veröffentlichungen und Drittmitteleinwerbung - ein Schwerpunkt bei den Forschungstätigkeiten liegt. Voraussetzung für den Ruf auf eine W2- oder W3-Hochschulprofessur stellt die Habilitation oder habilitationsäquivalente Leistung dar. Vor allem in den empirischen Wissenschaften wurde die monographische Habilitationsschrift in den letzten Jahren zunehmend abgelöst durch kumulative Habilitationen mittels einer Sammlung von Aufsätzen und Buchkapiteln. Voraussetzung für die Berufung auf eine Fachhochschulprofessur sind im Unterschied zur Hochschulprofessur Promotion und fünfjährige Tätigkeit in der Praxis.

An welchen Stellen im Berufungsverfahren werden Leistungskriterien wirksam? Wo liegen Einfallstore für nicht-meritokratische Mechanismen? Bei der Vorauswahl der zu Vorstellungsvorträgen einzuladenden Kandidaten werden die im Lebenslauf dokumentierten $\mathrm{Pu}-$ blikationen, Drittmitteleinwerbungen und bisherigen Tätigkeiten herangezogen. Unklar bleibt allerdings, wie stark diese Faktoren gewichtet werden, ob in allen Fällen eine Bewertung von Veröffentlichungen vorgenommen wird, z.B. eine Gewichtung nach der Art der Zeitschrift und der Zahl der Koautoren, und welche Rolle persönliche Kontakte von Mitgliedern der Berufungskommission zu Kandidaten oder deren Mentoren spielen, um überhaupt als möglicher Kandidat wahrgenommen zu werden. Schwierig zu bewerten sind didaktische Fähigkeiten, die sich allenfalls im Bewerbungsvortrag oder in bisweilen vorliegenden Lehrveranstaltungsbewertungen dokumentieren.

\section{Wissenschaftliche Produktivität}

Die Abgrenzung meritokratischer und nicht-meritokratischer Faktoren für wissenschaftliche Karrieren ist nicht ganz eindeutig. Daher wird die folgende Darstellung des Forschungsstandes unterteilt in wissenschaftliche Produktivität, die die meritokratischen Faktoren widerspiegeln soll, askriptive Merkmale als Synonym für nicht-meritokratische Mechanismen und Prozesse sowie institutionelle Faktoren, die z.B. den Einfluss der Reputation von Organisationen beschreiben. Insbesondere in den letzten beiden Gruppen ist die Abgrenzung von meri-

1) Zu Berufungsverfahren im internationalen Vergleich vgl. Schmitt et al. (2004). 
tokratischen und nicht-meritokratischen Mechanismen schwierig: Ist die Beschäftigung an einem renommierten Institut zufällig aus der individuellen Biographie (z.B. Nähe zum Wohnort) oder aufgrund einer bewussten karriereorientierten Wahl entstanden? Sind Beziehungen zu Kollegen funktional im Sinne sachlich erforderlicher Forschungskooperationen oder sind sie als Klüngel und Seilschaften zu bewerten, die Außenstehende ohne sachliche Begründung ausschließen? Die Diskussion zur Bedeutung von Schul- und Testleistungen bei der Zulassung an amerikanischen Universitäten zeigt darüber hinaus, dass selbst vermeintlich objektive Kriterien (wie ein Testergebnis) zu diskriminierenden Effekten beitragen können, wenn die Voraussetzungen für die Erlangung eines Testwerts sozial ungleich verteilt sind (Alon / Tienda 2007) oder die Präzision der Produktivitätsschätzung mittels Test mit sozialen Merkmalen korreliert (Phelps 1972a; 1972b). Im Folgenden wird der Forschungsstand zur Bedeutung der verschiedenen Kriterien zusammengefasst.

Wissenschaftliche Leistung gilt als universalistisches und legitimes Kriterium für die Rekrutierung von wissenschaftlichem Nachwuchs (vgl. etwa Krais 2000a; Paris 2003; Hartmann 2002, S.15f). Fraglich ist jedoch, welche Leistungen bei Bewerbern relevant sind, wie diese Leistungen messbar gemacht werden und wie Einzelleistungen in Relation bewertet werden. Drei zentrale Dimensionen können unterschieden werden: (1) Forschung und Publikation von Forschungsergebnissen, (2) Einwerbung von Drittmitteln und (3) Lehre. Fox (1992) untersuchte den Zusammenhang zwischen den unterschiedlichen Dimensionen akademischer Leistungen - Forschung, Lehre und Publikationsleistungen - in sozialwissenschaftlichen Fakultäten. Sie kommt zu dem Ergebnis, dass Personen mit vielen Publikationen auch viel in Forschung investieren, jedoch nicht in die Lehre. Gerade im universitären Kontext ist nach Reskin (1977) die im akademischen Berufsverlauf frühe Produktivität von Bedeutung.

Publikation ist nicht gleich Publikation. Die Qualität wissenschaftlicher Publikationen könnte sich beispielsweise bemessen an Innovativität, Kreativität, Einfluss auf künftige Forschung, Reichweite und Nützlichkeit der Ergebnisse. Diese Aufzählung deutet an, dass schon über die Kriterien nicht immer Einigkeit zu erzielen ist, ganz zu schweigen von der grundsätzlichen Möglichkeit und dem Aufwand, Publikationen nach diesen Kriterien zu bewerten. Aus diesem Grund versucht man, die Qualität von Publikationen anhand von Hilfskriterien zu bewerten. In der Bibliometrie wurden Publikations- und Zitationsmaße entwickelt, die personen- und institutionenbezogen publiziert werden. Mit der Erfindung des Science Citation Index (SCI) im Jahr 1961 wurde ein Messinstrument bereitgestellt, das davon geht man in der Regel aus - den öffentlich wahrgenommenen Beitrag eines Wissenschaftlers reliabel und valide misst (Cole / Cole 1973, S.21). Der Social Science Citation Index (SSCI) wird in der deutschsprachigen Soziologie einerseits als gut handhabbares Messinstrument verwendet (Litzenberger / Sternberg 2005; 2006) als auch als das kleinere Übel gegenüber alternativen Formen der Qualitätsmessung bewertet (Hirschauer 2004; 2005). Im Science Citation Index und Social Science Citation Index wird eine Auswahl von Zeitschriften, die bestimmten Qualitätsmaßstäben, wie peer-review Verfahren und Zitationshäufigkeit, genügen müssen, regelmäßig nach veröffentlichten Artikeln und Zitationen in diesen Artikeln ausgewertet. Kritisiert wird an diesen Indices, dass angloamerikanische Zeitschriften sowie Zeitschriften mit allgemeinen Themen gegenüber spezialisierten Zeitschriften dominieren und dass Monographien nicht berücksichtigt werden (Gläser 2006), obwohl sie in bestimmten Disziplinen wichtiger sind als Zeitschriftenartikel. Die Schreibweisen der Namen insbesondere mit Umlauten, die in der englischen Sprache nicht vorkommen - sind zudem oft fehlerhaft. Ehrenautorschaften schlagen auf Konten einzelner Forschungsmanager besonders zu Buche. Es erfolgt keine Gewichtung der Beiträge nach der Zahl der Koautoren oder der Länge der Beiträge. Die starke Resonanz in der scientific community bietet Anreize dafür, dass Autoren keine umfassenden Arbeiten schreiben, sondern die Projekte in kleinste, 
publizierbare Teile zerlegen, sich vornehmlich an den im Index aufgelisteten Zeitschriften orientieren und dass Zeitschriften Autoren dazu anhalten, frühere Publikationen in der Zeitschrift wieder zu zitieren, um den >journal impact factor $<$ hoch zu halten.

Heinze und Bauer (2007) untersuchten am Beispiel der Nanotechnologie die Merkmale kreativer Wissenschaftler und stellten die Frage, ob sich besonders innovative Wissenschaftler durch eine hohe Produktivität, Multidisziplinarität oder gemäß Burts Theorie der strukturellen Löcher durch eine Vermittlerrolle in einem wissenschaftlichen Netzwerk auszeichnen. Sie kommen zu dem Ergebnis, dass nicht die Quantität der Publikationen entscheidend ist, sondern die Fähigkeit, effektiv mit den Kollegen zu kommunizieren und ein breites, d.h. multidisziplinäres inhaltliches Spektrum abzudecken.

Ein Aspekt der wissenschaftlichen Leistungsbeurteilung ist die Wahrnehmung der Publikationen in der scientific community, die häufig anhand von Zitationsanalysen gemessen werden. Die Gegner dieser Messvariante plädieren dafür, nur die Zahl der zustimmenden Zitate zu berücksichtigen, die in extensiven Textanalysen ermittelt werden müsste (Endruweit 2002). Wie häufig jemand zitiert wird, hat nach Allison und Long (1987) einen positiven Effekt auf die Aufwärtsmobilität in Universitäten. Artikel von Frauen werden im Durchschnitt häufiger zitiert als diejenigen der Männer, wobei Männer eher auf die Quantität ihrer Literaturliste achten (Long 1992).

Dass Publikationen in referierten Zeitschriften relativ hoch bewertet werden, ist auf Selektionsprozesse zurückzuführen. In angesehenen Zeitschriften kann nur ein kleiner Anteil der eingereichten Artikel publiziert werden. Gutachter und Herausgeber können strenge Kriterien anlegen. Durchläuft eine Publikation keinen Begutachtungsprozess (wie z.B. bei Monographien oder häufig bei Beiträgen zu Sammelbänden), kann man nicht unbesehen von einer hohen Qualität ausgehen. Umso problematischer ist es, wenn die Begutachtungsverfahren nicht objektiv und valide messen und beispielsweise askriptive Merkmale der Autoren eine Rolle spielen. Bei nichtanonymen peer-review Verfahren gelingt es den Gutachtern oft nicht, geschlechtsunabhängig zu urteilen; vielmehr bevorzugen sie männliche Nachwuchsforscher (Wennerås / Wold 1997; 2000). Ein weiterer Kritikpunkt an der Bewertung durch peers ist die konservative Tendenz dieses Verfahrens (Heinze / Bauer 2007, S. 814). Neue und kreative Ideen werden möglicherweise nicht erkannt und gewürdigt, insbesondere wenn sie nicht konform zu den herrschenden Paradigmen sind.

Vielfach belegt ist der kumulative Effekt von wissenschaftlicher Produktivität, der auch unter dem Matthäuseffekt bzw. »Matthew Effect« bekannt ist (Merton 1968²; 1988; Allison / Stewart 1974; Allison et al. 1982). So wirkt sich nicht nur vergangene Produktivität positiv auf die gegenwärtige aus, sondern auch die Reputation eines Wissenschaftlers auf die Anerkennung und Honorierung seiner Leistung. ${ }^{3}$ Merton (1968) und Rose (1986) übertragen den Matthäuseffekt auch auf Institutionen. So erhalten gerade diejenigen Institutionen, die renommierte Wissenschaftler vorweisen können, besonders viel staatliche Forschungsförderung (Zuckerman 1967; 1993; Dey et al. 1997). In Bezug auf die Debatte um die deutschen Eliteuniversitäten gewinnt diese Diskussion an Brisanz.

2) Rossiter (1993) verweist darauf, dass der ursprüngliche Artikel von Merton (1968) eher anekdotischen Charakter hat als eine solide empirische Basis. Zudem zitiere er häufig die Dissertation seiner Mitarbeiterin und Ehefrau Harriet Zuckerman aus dem Jahr 1965. Rossiter deutet damit an, dass die Zuschreibung des Matthäuseffekts als Leistung Mertons vielleicht gerade eben diesen Effekt bestätigt. Zudem stellt Rossiter (1993) dem Matthäuseffekt den »Matilda Effect « gegenüber, der davon ausgeht, dass die herausragenden Leistungen von Frauen häufig ignoriert werden.

3) Analog dazu formulieren Preisendörfer und Voss (1988) die »Karrierezyklus-These«, wonach Personen mit fortgeschrittener Karriere auch mehr Personen kennen, die ihnen bei weiterer Arbeitsplatzsuche behilflich sein können. 
Die Kriterien, nach denen wissenschaftliche Produktivität bemessen wird, unterscheiden sich je nach Disziplin und sogar innerhalb von Fächern. ${ }^{4}$ Ebenso ist die Berufungsdauer stark abhängig von der Spezialisierung innerhalb eines Faches (für die Politikwissenschaft siehe Plümper und Schimmelfennig $2007^{5}$ ). Viele der bisher durchgeführten Studien sind blind gegenüber fächerspezifischen Charakteristika. Eine erfreuliche Ausnahme bildet die Studie von Enders und Bornmann (2001), die Doktoranden sechs unterschiedlicher Fächer untersucht hat. Ein Ergebnis dieser Studie besagt, dass die untersuchten Elektroingenieure zur Erlangung einer Professur mehr außeruniversitäre Praxiserfahrung sammeln müssen als Doktoranden aus den Fächern Biologie, Germanistik, Mathematik, Sozial- oder Wirtschaftswissenschaften (Enders / Bornmann 2001, S. 79). Dass Leistung - trotz ihrer zunächst universalistischen Natur - nicht mit einer einfachen Messlatte beurteilt werden kann, wird erst auf den zweiten Blick offenbar.

Im Rahmen einer Expertenbefragung mit 22 Vertretern aus vier Fachrichtungen, die im Rahmen eines DFG-Projekts durchgeführt wurde, konnte gezeigt werden, dass die wissenschaftlichen Standards in den einzelnen Fächern sehr unterschiedlich ausgelegt werden. ${ }^{6}$ Dabei werden fachspezifische Logiken angewandt. Im Maschinenbau spielen der Praxisbezug und die Bewährung in der Industrie eine zentrale Rolle für die Berufungsfähigkeit von Kandidaten. Rein akademische Meriten werden nicht so wichtig genommen, sodass häufig eine Habilitation nicht als Voraussetzung angesehen wird. In der Jurisprudenz hingegen ist die Qualifikationsleistung in Gestalt von Staatsexamina, Promotionsnote und Habilitation das zentrale Kriterium. Diese Qualifikationsleistungen müssen an angesehenen Instituten erworben sein, um entsprechende Berufungschancen zu generieren. Professionsangehörige verfügen über eine »mental map«, die genaue Auskunft darüber gibt, wo die Koryphäen der verschiedenen Rechtsgebiete sitzen. Flankiert wird dieses System durch ein »tacit knowledge « (Polanyi 1966) über geeignete, d.h. hoch reputierliche, juristische Fachverlage und subtile Markierungen des »Stallgeruchs«, z.B. in Form der Schriftart, in der publiziert wird. Die Rechtswissenschaft zeichnet sich also durch eine Kombination von universalistischen und partikularistischen Prinzipien aus. In der Mathematik sind Publikationen in international renommierten Fachzeitschriften das zentrale Kriterium. Darüber hinaus finden sich innerhalb der Disziplin erhebliche Differenzierungen, die vor allem entlang der Trennungslinie zwischen rein theoretischen Spezialisierungen und angewandten Bereichen verlaufen. Während die Zahl der Professuren im Bereich der Theorie gering ist, scheint der praxisorientierte Bereich bessere Möglichkeiten zu bieten. Hier sind auch die Möglichkeiten zur Einwerbung von Drittmitteln und deren entsprechende Wertschätzung höher. In der Soziologie pendeln die Ergebnisse zwischen einer Betonung von quantifizierbarer Publikationsleistung (wobei deutschsprachige Veröffentlichungen zwar quantitativ dominieren, internationale Veröffentlichungen aber höher anerkannt werden), der Erkenntnis, dass dieses Kriterium auch gewisse Validitätsprobleme aufweisen kann, und der empirischen Erkenntnis, dass sich im konkreten Verfahren bisweilen sehr partikularistische Kriterien durchsetzen. Zusammenfassend kann man auch aus den Ergebnissen dieser Expertengespräche die Hypothese folgern, je standardisierter die Leistungsmessung in einem Fach, desto weniger bedeutsam sind persönliche Merkmale bzw. desto geringer ist die Diskriminierung entlang dieser Merkmale. Diese Hypothese sollte in weiteren empirischen Studien geprüft werden.

4) Dieses Problem spielt auch in den immer häufiger durchgeführten Evaluationen und Rankings von Fächern eine Rolle. Das CHE Forschungsranking 2006 berücksichtigt für die verschiedenen Fächer unterschiedliche Publikationsarten und bibliographische Quellen (Berghoff et al. 2006).

5) Von weiteren Ergebnissen der Studie von Plümper und Schimmelfennig (2007) zu berichten ist wenig sinnvoll, da die methodische Vorgehensweise dem Forschungsgegenstand unangemessen erscheint (ausführlicher dazu Gross und Jungbauer-Gans 2007).

6) DFG-Projekt JU 414/5-1; Vgl. Gross et al. 2008 
Zunehmend Beachtung findet die Einwerbung von Drittmitteln. Die einfache Möglichkeit der Quantifizierung führt dazu, dass Drittmittel immer häufiger als Indikator für exzellente Forschung herangezogen werden (Münch 2006). Dabei unterscheiden sich jedoch die Möglichkeiten und die Notwendigkeit des Einwerbens von Drittmitteln erheblich zwischen den Fächern und sogar zwischen den Teildisziplinen innerhalb der Fächer (Neidhardt 2006; Gerhards 2002; Hartmann 1988). Die durchschnittliche Bewilligungssumme im Halbjahr pro Professor/in für die Jahre 1983 bis 1988 beträgt beispielsweise im Maschinenbau / Verfahrenstechnik DM 96.000.-, in den Sozialwissenschaften DM 15.000.- und in den Rechtswissenschaften nur rund DM 2.000.- (Hornbostel 1997, S. 233). Auch die Quellen der Drittmittel unterscheiden sich erheblich. Die DFG spielt in der Informatik, Mathematik und Physik die größte Rolle, Stiftungen in der Rechtswissenschaft. Die angewandten Fächer nehmen häufiger Industrieaufträge an (Hornbostel 2001). Grundsätzlich wird darüber diskutiert, ob der Umfang der Drittmittel auch mit dem Forschungsoutput beispielsweise in Form von (hochrangigen) Publikationen korreliert. Zumindest für die Soziologie stellt Münch (2006) fest, dass es keinen signifikanten Zusammenhang zwischen der Höhe der eingeworbenen Drittmittel und der Anzahl der Publikationen pro Professor gibt.

Eine weitere Facette akademischer Leistung sind Lehre und didaktische Fähigkeiten. Dabei ist zu konstatieren, dass die Beachtung von didaktischen Fähigkeiten bei Berufungen erst im Verlauf der letzten Dekade zunehmend an Bedeutung gewann. Studentische Lehrevaluationen scheinen jedoch nicht das Mittel der Wahl zu sein, um didaktische Fähigkeiten zu bewerten. Ergebnisse einschlägiger Studien zeigen, dass die Motivlage der Studierenden (intrinsisch vs. extrinsisch) die Bewertung der Lehrleistung erheblich beeinflusst (Kromrey 1995). Pflichtveranstaltungen werden schlechter bewertet als Wahlveranstaltungen, große Veranstaltungen schlechter als kleine, ältere Studierende werten weniger kritisch als jüngere, Veranstaltungen mit abstrakten, theoretischen und methodischen Inhalten werden schlechter bewertet als beschreibende. Auch die Raumverhältnisse wie Licht, Temperatur oder Störung durch Lärm etc. spielen eine Rolle (Kromrey 2004). Klein und Rosar (2006) konnten sogar einen positiven Effekt der physischen Attraktivität der Dozenten auf die Lehrevaluationen nachweisen, womit sie Ergebnisse von Hamermesh und Parker (2006) für Deutschland replizieren können.

\section{Askriptive Merkmale}

Dass leistungsbezogene Kriterien die ausschlaggebenden und einzigen Faktoren sind, die den Verlauf von wissenschaftlichen Karrieren bestimmen, galt lange Zeit als unhinterfragte Tatsache. Erst die Forschung zur Benachteiligung von Frauen oder Minoritäten im Hochschulwesen und die Analyse der Zugangschancen zu gesellschaftlichen Elitepositionen (Hartmann 1996; 2002; Hartmann / Kopp 2001) haben das Augenmerk auf Faktoren gelenkt, die wenig mit meritokratischen Prinzipien zu tun haben.

Auf europäischer Ebene sind Frauen bei den Studierenden und Hochschulabsolventen mittlerweile überrepräsentiert (European Commission 2006, S. 55). Auf der Stufe der Doktoranden ist der Anteil der Männer etwas höher, während sich die Schere drastisch öffnet, je höher die Besoldungsstufe auf der Ebene der Professoren ist. Dabei gibt es erhebliche Variationen zwischen den Ländern. Deutschland gehört mit einem Frauenanteil von 29,2\% auf allen akademischen Qualifikationsstufen zu den Schlusslichtern. Dabei scheint aktuell die Chance auf eine Berufung unter Habilitierten nicht abhängig vom Geschlecht zu sein (Löther 2006), sodass eine weitere Erhöhung des Frauenanteils zu erwarten ist.

Frauen sind weniger produktiv als Männer (Long/Fox 1995), weil sie seltener über Ressourcen und Positionen verfügen, die einer höheren Produktivität förderlich sind (Leahey 2007). Ein Großteil der Produktivitätsunterschiede zwischen Frauen und Männern in der 
Wissenschaft kann erklärt werden durch persönliche Merkmale, strukturelle Positionen, wie z.B. die Art der beschäftigenden Organisation, Umfang der Drittmittel, Zahl der Forschungsmitarbeiter, begrenzte Lehr- und administrative Verpflichtungen, unterstützende soziale Netzwerke und Mitarbeit in Forschungskooperationen und den Familienstand (Fox 2005; Xie / Shauman 1998; vgl. zusammenfassend Leahey 2007). Die Produktivitätsunterschiede zwischen Frauen und Männern nahmen im Zeitverlauf ab (Xie / Shauman 1998). Welche Faktoren sich auf das Einkommen von Frauen und Männern in der Wissenschaft auswirken, wurde von Leahey (2007) untersucht. Sie stellte dabei fest, dass sich wissenschaftliche Produktivität nur indirekt auf das Einkommen amerikanischer Wissenschaftler auswirkt. Frauen spezialisieren sich weniger auf bestimmte Forschungsfelder und sind deshalb weniger sichtbar in ihrer wissenschaftlichen Community. Die Sichtbarkeit vor allem erklärt die unterschiedliche Höhe des Einkommens (Leahey 2007).

Achatz und Hinz (2001) belegen mit Paneldaten von Forschungsinstitutionen, dass mit einer Verschlechterung der Randbedingungen im Sinne eines höheren Anteils befristeter Stellen der Frauenanteil steigt. Dieser Sachverhalt steht im Einklang mit den Ergebnissen von Bochow und Joas (1987), die als Gründe für das Ausscheiden aus einer universitären Laufbahn bei Männern häufiger als bei Frauen folgende Gründe ermitteln: »Verbesserung des Gehalts«, »Verbesserung der Arbeitsmöglichkeiten« und »Aussicht auf eine Leitungsposition« (Bochow / Joas 1987, S. 54). Die Karriereorientierungen von Männern und Frauen unterscheiden sich dabei nach Abele (1994) nicht. Die geschlechtsabhängigen Bewilligungschancen von DFG-Forschungsanträgen wurden von Allmendinger und Hinz (2002) untersucht. Sie kommen zu dem Ergebnis, dass die deutlich schlechteren Bewilligungschancen von Frauen zum Großteil durch die Konzentration auf bestimmte Forschungsinhalte und die dadurch entstehende Konkurrenz erklärt werden können.

Zudem erfahren Frauen und Männer unterschiedliche Unterstützung durch den Lebenspartner. Hochschullehrer sind deutlich häufiger verheiratet als Hochschullehrerinnen (Bauer 1986). Eine mögliche Interaktion in der gemeinsamen Wirkung von Geschlecht und Familienstand auf das Einkommen ist unter dem Begriff »marriage premium « bekannt (Kermit 1995). Demnach verdienen verheiratete Männer mehr als ledige, während der Familienstand sich bei Frauen nicht auf Einkommen und Berufsstatus auswirkt. Astin und Bayer (1979) konnten nicht nur den »marriage premium effect « für Wissenschaftler belegen, sondern auch, dass verheiratete Wissenschaftlerinnen weniger verdienen und statustiefere Beschäftigungen haben als ihre ledigen Kolleginnen. Den karriereförderlichen Effekt von externer sozialer Unterstützung bei Männern und deren Ausbleiben bei Frauen konnten auch Fuchs et al. (2001) für Wissenschaftlerinnen und Wissenschaftler der Max-Planck Gesellschaft zeigen.

Engler (2000; 2001) beschreibt die Vorrangstellung der wissenschaftlichen Persönlichkeit in Berufungsverfahren und argumentiert, dass diese nun mal männlich sei und die Professorenstellen somit auch unter Männern ausgehandelt und erkämpft werden (zu der Funktionsweise dieser Machtspiele siehe auch Zimmermann 2000). Die Wahrnehmung dieses Kampfes (oder in Anlehnung an Bourdieu: dieses »Spiels«) aus Perspektive der Akademikerinnen beschreibt Krais (2000b) wie folgt:

»Sie machen sich lustig über die 'Hahnenkämpfe' unter Männern, sind peinlich berührt von deren 'substanzloser Selbstdarstellung' oder leiden unter deren 'killing instinct' und der Anforderung, sich ebenso zu verhalten.« (Krais 2000b, S. 47)

Paradoxerweise beschreiben zwar viele Akademikerinnen geschlechtsspezifische Diskriminierung an der Universität, behaupten jedoch, persönlich davon nicht betroffen zu sein (Krais 2000c; Wetterer 1986). Nach Krimmer et al. (2003, S. 3) ist die Situation von Frauen an der Hochschule von »horizontaler, vertikaler und kontraktueller Segregation« gekenn- 
zeichnet. Das heißt, die Professorinnen konzentrieren sich auf bestimmte Fächer, niedrigere Positionen und befristete Arbeitsverhältnisse (zusammenfassend auch Teichler 1996). Stebut und Wimbauer (2003) veranschaulichen die kurze Aufenthaltsdauer der Frauen in Forschungsorganisationen mit der Metapher der »Drehtür «. ${ }^{7}$ Die hohe Fluktuation bei Frauen in der Wissenschaft nimmt dabei mit höheren Frauenanteilen zu, was die These bestätigt, dass mit dem Anwachsen von Minoritäten auch der Wettkampf innerhalb dieser Gruppen steigt. Erst bei einem Frauenanteil von 35-40 \% nimmt die Fluktuation wieder ab (Tolbert et al. 1995).

Broder (1993) stellte im Zuge einer Längsschnittuntersuchung mit Wirtschaftswissenschaftlern größere geschlechtsspezifische Gehalts- und Statusunterschiede in den älteren Kohorten als in den jüngeren fest und auch Clark und Corcoran (1986) sprechen von einer Abnahme der feindseligen Haltung gegenüber Akademikerinnen.

Während das Thema »Chancen von Migrantenkindern im Bildungssystem « spätestens seit der Veröffentlichung der PISA-Ergebnisse in der deutschen bildungssoziologischen Diskussion angekommen ist, scheinen die Chancen von Mitgliedern ethnischer Minderheiten oder von Ausländern in Hochschule und Forschung - im Gegensatz zu den USA - bislang keine Rolle zu spielen. Umso dringender sollte diese Frage auch für Deutschland untersucht werden. Eine amerikanische Studie untersuchte die Einkommensunterschiede in der Wissenschaft bei verschiedenen ethnischen Gruppen und kam zu dem Ergebnis, dass Angehörige von Minoritäten (mit Ausnahme von lateinamerikanischen Frauen) sogar höhere Einkommen haben als weiße Männer, während weiße Frauen im Durchschnitt ein niedrigeres Einkommen aufweisen (Monks / Robinson 2000). Zugewanderte Personen und Ausländer haben ein niedrigeres Einkommen als U.S.-Bürger.

Die Chance auf eine Spitzenposition in der Wirtschaft steigt nach Hartmann und Kopp (2001) mit der sozialen Herkunft. Bei Spitzenpositionen in der Wissenschaft (Professuren) sei dieser Effekt auch bemerkbar, fällt allerdings nicht so deutlich aus wie in der Wirtschaft (Hartmann 2002). Rothböck et al. (1999) diagnostizieren anhand einer Schweizer Studie gerade der wissenschaftlichen Elite - mehr noch als der wirtschaftlichen oder politischen Elite - eine besondere Verschlossenheit gegenüber Personen aus der Unter- und Mittelschicht. Die soziale Herkunft wirkt sich nicht nur über den Umweg der besonders hohen Bildungsanforderungen aus, die die Oberschicht leichter erfüllen kann, sondern auch direkt über einen schichtspezifischen Habitus. Wissenschaftlerinnen kommen häufiger aus Akademikerelternhäusern (Bauer et al. 1993, S. 105). Frauen aus der Unterschicht sind durch homosoziale Rekrutierungsstrategien in doppeltem Sinne benachteiligt (Hasenjürgen 1996, S. 270f), da sie sich sowohl an das männlich geprägte als auch an das bildungsbürgerliche Milieu anpassen müssen.

Die Bedeutung der Netzwerkeinbindung für die erfolgreiche Jobsuche wird spätestens seit dem klassischen Werk »Getting a Job « von Granovetter (1995[1974]) nicht mehr in Frage gestellt. Schwache Beziehungen sind insbesondere dann von Nutzen, wenn es um Informationsfluss geht (Granovetter 1973; 1983), sie helfen jedoch nach Wegener (1991) nur Personen mit einem vorherigen statushohen Arbeitsplatz. Mouw (2003) stellt die Kausalität auf den Kopf und argumentiert, dass ähnliche Personen mit höherer Wahrscheinlichkeit Freunde werden und sich nicht das Sozialkapital auf die berufliche Position auswirkt. Die informellen Informationskanäle sind jedoch nach de Graf und Flap (1988) auf dem US-amerikanischen Arbeitsmarkt von größerer Bedeutung als auf dem deutschen und niederländischen Arbeitsmarkt.

7) Sonnert (1995) sieht hierbei eine Analogie zu Simmels Fremden, der bekanntlich nicht nur heute kommt und morgen geht, sondern eben bleibt. Mit anderen Worten: Ein kurzes Gastspiel ist noch kein Problem, aber wenn der »Gast « beschließt zu bleiben, dann treten Probleme auf. 
Krackhardt (1992) dagegen verweist auf die Bedeutung von starken Beziehungen innerhalb des Unternehmens, die sich idealerweise über das Unternehmen verteilen. Der Verbleib von Wissenschaftlerinnen in renommierten Forschungsinstituten wie etwa dem Max-Planck-Institut bedingt sich stark durch die Einbindung in Netzwerke innerhalb von Forschungsinstitutionen oder der scientific community im Allgemeinen (Allmendinger et al. 1998).

Long (1990) und Long und McGinnis (1985) argumentieren, dass der entscheidende Einfluss auf die Produktivität von der Zusammenarbeit mit einem Mentor ausgeht (vgl. auch Allmendinger et al. 1998). Soziale Unterstützung und daraus folgende Produktivität bedingen sich also gegenseitig. Lang und Neyer (2004) konnten anhand einer Studie mit Psychologen aufzeigen, dass die Produktivität des Erstgutachters die Wahrscheinlichkeit, eine Anstellung an der Universität zu erhalten, erhöht. Eine sehr hohe Leistung ist allerdings nicht unbedingt Voraussetzung, um als Zögling von einem Mentor auserkoren zu werden (Long / McGinnis 1985). Die Förderung durch einen Mentor lässt bei Frauen allerdings nach, wenn sie kleine Kinder haben; bei Männern wird die Zusammenarbeit mit dem Mentor hingegen nicht durch Kinder beeinflusst (Long 1990). Zahlreiche Arbeiten haben gezeigt, dass die nach Geschlecht variierenden Karrierechancen auch durch unterschiedliche Einbindung in Netzwerke allgemein erklärt werden können (Burt 1998; Beggs / Hurlbert 1997; Ibarra 1992). Keine Karrierenachteile erfahren Frauen, die eine stabile Mentor-Mentee-Beziehung zu einem männlichen Mentor aufweisen (Burt 1998). Weniger Unterstützung durch einen Mentor erfahren Personen, die eine sehr weibliche oder sehr männliche Geschlechtsrollenorientierung aufweisen (Scandura / Ragins 1993). Die grundlegendste Kritik am Wirken universalistischer Prinzipien wird von Long et al. (1979) ins Feld geführt: Wenn man auf wissenschaftliche Herkunft und das Prestige des Mentors kontrolliert, hat die vergangene Produktivität keinen Effekt auf das Prestige der ersten akademischen Anstellung.

Nur ein sehr geringer Teil der im Wissenschaftssystem anerkannten Leistungen wird von einem Forscher alleine bewerkstelligt (dazu gehören größtenteils Lehrleistungen, die einen nachrangigen Stellenwert besitzen). Für den weitaus bedeutenderen Teil - Publikationsleistungen und Einwerben von Drittmitteln - spielen soziale Kontakte eine große Rolle und sind oft nicht mehr eindeutig von produktiven Leistungen zu trennen. Die Wirkung sozialer Kontakte bei der Besetzung von Stellen, der Annahme von Artikeln bei Zeitschriften oder dem gemeinsamen Einwerben von Drittmitteln wird allgemein sehr zwiespältig gesehen. Zum einen wird die fachliche Unterstützung eines Mentors als wichtige Komponente der persönlichen fachlichen Weiterentwicklung gesehen. Zum anderen gelten Entscheidungen in Abhängigkeit von sozialen Beziehungen bei Leistungsbeurteilungen, wie etwa der Annahme oder Ablehnung von Manuskripten, im peer-review Verfahren oder von Kandidaten bei Berufungsentscheidungen als illegitim.

Die Frage, in welchem Alter Wissenschaftler im Durchschnitt ihre produktivste und innovativste Phase haben, war bereits der Gegenstand von Studien. Cole (1979) stellte einen umgekehrt u-förmigen Zusammenhang zwischen dem Lebensalter und der Quantität und Qualität von Publikationen fest. Die produktivste Phase liegt im Alter von 30 bis 40 Jahren, wobei es erhebliche Differenzen zwischen den Fächern gibt. In der Mathematik liegt diese Phase beispielsweise erheblich niedriger als in der Soziologie. Die auftretenden empirischen Muster erklärt Cole (1979) mit dem wissenschaftlichen Anerkennungssystem. Stephan und Levin (1992) stellten fest, dass Nobelpreisträger ihre Bahn brechenden Arbeiten etwa im Alter von 40 Jahren durchführten. Ist der wissenschaftliche Zenit überschritten, sinkt die Produktivität von Forschern im Durchschnitt. Alarmierend wird dieser Befund, wenn sich die Altersstruktur der Wissenschaftler ändert: Wird das Durchschnittsalter höher, reduzieren sich die zu erwartenden Innovationen (Stephan / Levin 1992). In einer verfeinerten Analyse, die nicht das Lebensalter, sondern den Zeitpunkt der Promotion als Normierungspunkt für das Alter verwendet, zeigte sich, dass ein Polynom Fünfter Ordnung, also eine zwei-gipfelige Verteilung mit einem höheren ersten Gipfel, das empirische Muster besser beschreibt als ein quadratischer Term (Goodwin / Sauer 1995). 
Die ältere Kohorte kann zudem die Arbeit der Jüngeren behindern, wenn ihre Dominanz dazu führt, dass neue Ansätze und Ideen abgelehnt werden (Stephan / Levin 1992). Die Altersstruktur beeinflusst auch die Zahl an neu zu besetzenden Stellen und damit die beruflichen Chancen der Nachwuchswissenschaftler. Dieses Problem wurde in den 80er Jahren in Deutschland offensichtlich, als beim Ausbau der Universitäten in den 60er und 70er Jahren eine relativ junge Kohorte berufen wurde, die ihre Stellen über 25 bis 30 Jahre behielt. Die in den 80er Jahren Habilitierten hatten nach der Vollendung des Ausbaus wesentlich weniger Opportunitäten als die Generation der 70er Jahre. Erst mit der Reform des Hochschulwesens in Ostdeutschland (Schluchter 1996) änderte sich diese Situation. Haben Nachwuchswissenschaftler wenige Chancen auf eine Beschäftigung in Universitäten, führt dies in der Regel zu einem Qualitätsverlust, weil besonders fähige Personen leichter alternative Beschäftigungen außerhalb von Hochschule und Forschung finden (Stephan / Levin 1992).

Auch zwischen dem bereits angesprochenen Matthäus-Effekt und dem Alter gibt es Zusammenhänge, da der Erwerb akademischer Meriten Zeit erfordert. Der Matthäus-Effekt führt u.a. aber dazu, dass die erworbene Reputation nicht entwertet wird, selbst wenn Produktivitätskennziffern absinken. So konnte Zuckerman (1968) zeigen, dass die Produktivität von Nobelpreisträgern nach Erhalt des Preises absinkt. Mit dem umgekehrt u-förmigen Produktivitätsmuster ist auch die These eines Rollenwandels vereinbar. Ist ein Erfolg erzielt, verändert sich die Rolle vom innovativen Primärforscher zum Forschungsmanager. Belegt werden kann diese These durch das Ergebnis, dass mit zunehmendem Alter der Anteil administrativer Tätigkeiten ansteigt (Merton / Zuckerman 1973) und dass Nobelpreisträger bei späteren Publikationen in der Autorenrangfolge häufig - möglicherweise in ihrer Rolle als Projektleiter - den letzten Platz einnehmen, was auch als »noblesse oblige « interpretiert wird (Zuckerman 1968).

\section{Institutionelle Faktoren}

Ob Universitäten mit hohem Prestige gute Wissenschaftler anziehen oder renommierte Institute gute Forschung ermöglichen, ist ein häufig diskutiertes Thema. Forschungsergebnisse zeigen, dass sich eher die Institution auf die Produktivität auswirkt als umgekehrt (Crane 1965; Long 1978; Long / McGinnis 1981; Allison / Long 1990). Crane (1965) führt diesen Effekt darauf zurück, dass renommierte Institute den Kontakt mit den jeweiligen Koryphäen des Faches erleichtern. Allein die Größe des Instituts (gemessen an der Anzahl promovierter Kollegen), an dem man promoviert, wirkt sich positiv auf die Wahrscheinlichkeit aus, ProfessorIn zu werden (Lang / Neyer 2004). Zudem haben exklusive Bildungseinrichtungen einen Signaleffekt (Spence 1973). In Kombination mit besserer Ausstattung an materiellen Ressourcen eignet sich diese Situation zur Reproduktion sozialer Strukturen im Hochschulwesen (Bourdieu et al. 1981[1971]; Hartmann 1999; 2001a; 2001b). In Deutschland ist dieser Effekt jedoch gering, verglichen mit Frankreich (Hartmann 1997), zumal man lange Zeit das Ziel einer gleichwertigen Ausbildung an allen Hochschulen verfolgte. Möglicherweise ändert sich die Situation in Deutschland auch mit den neu definierten und staatlich geförderten Eliteuniversitäten in München und Karlsruhe (man denke an einen Matthäus-Effekt auf Institutionenebene). Bisher besitzen eher Max-Planck-Institute eine exquisite Reputation im deutschen Wissenschaftssystem, deren Mitarbeiter noch dazu von Lehrverpflichtungen entlastet sind (Joas 1993). Dass Frauen zumindest in den USA seltener an prestigereichen Institutionen zu finden sind (Bielby 2000), könnte auch ein Grund für die schlechteren Karrierechancen der Frauen sein.

Wer an einem großen, renommierten Institut promoviert oder habilitiert hat, hat nicht nur mit hoher Wahrscheinlichkeit ein größeres fachliches Netzwerk, sondern kennt auch mit höherer Wahrscheinlichkeit die dort ansässigen Größen des Faches und musste sich im Habilitationsverfahren dem kritischen Urteil derer unterwerfen. Daher ist der Reputationseffekt von Institutionen nicht nur als diskriminierender Faktor zu bewerten, sondern kann auch Produktivitätsvorteile mit sich bringen. 


\section{Resümee}

In einem einleitenden Abschnitt wurde die Bedeutung von Normen und Werten für die Produktion wissenschaftlichen Wissens aufgezeigt. In diesem Text gingen wir der Frage nach, welche Mechanismen in wissenschaftlichen Karrieren eine Rolle spielen. Wir verfolgen damit eine analytische Soziologie, die sich zum Ziel gesetzt hat, die sozialen Mechanismen zu untersuchen, die bestimmte Muster und Prozesse erklären können (Hedström 2005; Hedström / Swedberg 1998; Maurer 2006). Soziale Prozesse werden durch das Handeln von Individuen erklärt (Haller 2003, S. 613), die sich rational im Sinne einer kognitiven Rationalität (Boudon 1998) verhalten. Dieser Ansatz rekurriert auf eine Handlungstheorie, die die Wünsche, Überzeugungen und Opportunitäten von Akteuren berücksichtigt. Zentral ist die Forderung, dass der untersuchte Prozess mit einer nachvollziehbaren Logik beschrieben werden muss (also keine »black box« darstellt). Die untersuchten Prozesse können im Zusammenwirken der beteiligten Akteure unintendierte Folgen haben (Haller 2003).

Im Zuge der Argumentation dieses Textes wurden die von Robert K. Merton postulierten Werte des wissenschaftlichen Ethos in Bezug gesetzt zu empirisch vorfindbaren Bewertungsmaßstäben. Dies geschah vor dem Hintergrund der These, dass die Merton'schen Normen geeignet sind, die Produktivität und Innovativität zu fördern. In diesem Zusammenhang ist vor allem der Wert des Universalismus relevant, der die meritokratischen Merkmale von Wissenschaft und Forschung in den Vordergrund stellt. In Bezug auf die Auswahl von Personen für wissenschaftliche Tätigkeiten legt Merton nahe, dass sie vor allen Dingen nach ihrem »Talent « - oder allgemeiner gesprochen nach Leistungskriterien - selektiert werden sollten. Wie diese Kriterien exakt zu operationalisieren sind, kann jedoch über verschiedene Disziplinen hinweg und sogar innerhalb von Disziplinen nicht so einfach entschieden werden. Dass die Beurteilung von Leistung nicht mit einer einfachen Messlatte erfolgen kann, war die Lehre aus dem vierten Abschnitt dieses Textes.

In dem oben angesprochenen Forschungsprojekt, in dessen Rahmen dieser Beitrag entstand, wird die relative Bedeutung der verschiedenen Leistungsindikatoren, der sozialen Einbindung, des Hochschulortes und der askriptiven Merkmale anhand von individuellen Karriereverläufen von Habilitierten der verschiedenen Fächer untersucht. Ist die Stufe der Habilitation erreicht, sind Prozesse der Selbstselektion, wie sie häufig zur Begründung des auf dem Weg der verschiedenen Qualifikationsstufen stark abnehmenden Frauenanteils herangezogen werden, weitgehend auszuschließen. Eine Antwort auf die Frage, welche Bedeutung askriptive Merkmale haben, ist demzufolge auch ein Indikator für das Ausmaß an Diskriminierung in wissenschaftlichen Karrieren.

Universalistische Normen werden in Zweifel gezogen angesichts der empirisch zu beobachtenden Muster der Benachteiligung von Frauen, ethnischen Minoritäten und Mitglieder niedrigerer sozialer Klassen sowie aufgrund der Bedeutung sozialer Netzwerke. Zwei Einschränkungen müssen jedoch gemacht werden. (1) Wie überzeugend die empirische Evidenz im Hinblick auf diese Muster tatsächlich ist, muss bei einzelnen Merkmalen noch systematisch untersucht werden. (2) Ob diese Muster tatsächlich für Leistungen negierende, diskriminierende Auswahlprozesse sprechen, ist nicht für alle Merkmale eindeutig. Eine bessere Einbindung in soziale Netzwerke könnte zu einer nicht zu rechtfertigenden Bevorzugung führen. Sie könnte aber auch eine Voraussetzung für die Beteiligung an größeren Forschungskooperationen in immer wichtiger werdenden interdisziplinären Forschungsclustern darstellen. Daher kann man soziale Netzwerke nicht unbedingt als »Klüngel « abstempeln, sondern muss sie unter Umständen auch als »soziales Kapital « ansehen, das in der weiteren Arbeit investiert wird und sich mitunter aus den social skills der Akteure speist. Ähnliche Ambivalenzen in der Beurteilung treten bei institutionellen Faktoren auf. Im Einzelfall kann es von biografischen Zufällen abhängen, in einem renommierten Institut zu studieren oder eine Qualifikationsstelle zu bekom- 
men. Trotzdem könnten positive, d.h. produktivitätsfördernde Effekte auftreten durch die vielschichtige Förderung durch Mentoren und Personen, denen das Institut seinen Ruf verdankt.

Dieser Beitrag hatte auch das Ziel, einen Teil des »tacit knowledge « über die Faktoren, die in wissenschaftlichen Karrieren ausschlaggebend sein können, offensichtlich zu machen. NachwuchswissenschaftlerInnen sollten sich klar werden über die Kriterien, nach denen in ihrer jeweiligen Disziplin Leistung bemessen wird: Welche Form der Publikation wird höher bewertet? Wer sind die wichtigen Akteure in meinem Forschungsfeld? Wie verlaufen Verbindungen und welche Struktur haben die bestehenden sozialen Netzwerke?

Abschließend soll darüber hinaus betont werden, dass die aktuellen Entwicklungen in Hochschulen und Forschung dazu geeignet sind, den Leistungskriterien eine breitere Basis zu verschaffen. Es ist also zu erwarten, dass die Bewertung wissenschaftlicher Leistung stärker über die Fächer hinweg standardisiert wird. Im Hinblick auf Diskriminierungsprozesse könnte dies zu gerechteren Mechanismen der Personalrekrutierung und im Hinblick auf Qualitätsstandards zu höheren Leistungsanreizen führen. Dies würde aber - unter der plausiblen Annahme, das sich quantitative Leistungsmessungen durchsetzen werden - bedeuten, dass sich vor allem die geisteswissenschaftliche Disziplinen, in denen offensichtlich Monographien nach wie vor hoch geschätzt werden, stärker am Publikationshabitus und den Bewertungsschemata der naturwissenschaftlichen Fächer orientieren müssen. Dies könnte aber auch bedeuten, dass Fächer, die diesen Wandel - oft aus guten inhaltlichen Gründen - nicht mitmachen wollen, langfristig ins Hintertreffen geraten, beispielsweise wenn es um die universitätsinterne, indikatorgestützte Verteilung von Haushaltsmitteln oder von Leistungsbezügen bei W-Besoldungen geht.

\section{Literatur}

Abele, A. (1994): Karriereorientierungen angehender Akademikerinnen und Akademiker, Bielefeld.

Achatz, J. / Hinz, T. (2001): Wandel einer Wissenschaftsorganisation und die Integration von Frauen, in: Zeitschrift für Soziologie 30, S. 323-340.

Allison, P. D. / Long, J. S. (1987): Interuniversity Mobility of Academic Scientists, in: American Sociological Review 52, S. 643-652.

Allison, P. D. / Long, J. S. (1990): Departmental Effects on Scientific Productivity, in: American Sociological Review 55, S. 469-478.

Allison, P. D. / Long, J. S. / Kauze, T.K. (1982): Cumulative Advantage and Inequality in Science, in: American Sociological Review 47, S. 615-625.

Allison, P. D. / Stewart, J. A. (1974): Productivity Differences among Scientists: Evidence for Accumulative Advantage, in: American Sociological Review 39, S. 596-606.

Allmendinger, J. / Hinz, T. (2002): Programmierte (Un-)Gleichheit? Geschlechtsspezifische Chancen bei der Bewilligung von Forschungsanträgen, in: Zeitschrift für Soziologie 31, S. 275-293.

Allmendinger, J. / von Stebut, N. / Fuchs, S. / Hornung, M. (1998): Berufliche Werdegänge von Wissenschaftlerinnen in der Max-Planck-Gesellschaft, in: INIFES, ISF, SÖSTRA (Hrsg.), Erwerbsarbeit und Erwerbsbevölkerung im Wandel. Anpassungsprobleme einer alternden Gesellschaft, Frankfurt-New York, S. 143-152.

Alon, S. / Tienda, M. (2007): Diversity, Opportunity, and Shifting Meritocracy in Higher Education, in: American Sociological Review 72, S. 487-511.

Astin, H. S. / Bayer, A. E. (1979): Pervasive Sex Differences in the Academic Reward System: Scholarship, Marriage, and What Else, in: D.R. Lewis / W.E. Becker Jr. (Hrsg.), Academic Rewards in Higher Education, Cambridge, S. 211-229.

Bauer, A. (1986): Wissenschaft und Hochschule als Beruf: Fragestellungen - Methoden - erste Ergebnisse, in: B. Clemens / S. Metz-Göckel / A. Neusel / B. Port (Hrsg.), Töchter der Alma Mater, Frankfurt, S. $135-152$. 
Bauer, A. / Gröning, K. / Hartmann, S. / Hausen, G. (1993): Die Regel der Ausnahme: Hochschulfrauen, Frankfurt.

Beggs, J. J. / Hurlbert, J. S. (1997): The Social Context of Men's and Woman's Job Search Ties: Membership in Voluntary Organizations, Social Resources, and Job Search Outcomes, in: Sociological Perspectives 40, S. 601-622.

Berghoff, S. / Federkeil, G. / Giebisch, P. / Hachmeister, C.-D. / Hennings, M. / Müller-Böling, D. (2006): Das CHE-Forschungsranking 2006, Arbeitspapier Nr. 79, Centrum für Hochschulentwicklung, Gütersloh.

Bielby, W.T. (2000): Geschlecht und Karriere: Ist die Wissenschaft ein Sonderfall? in: B. Krais (Hrsg.), Wissenschaftskultur und Geschlechterordnung, Frankfurt-New York, S. 55-81.

Bochow, M. / Joas, H. (1987): Wissenschaft und Karriere. Der berufliche Verbleib des akademischen Mittelbaus, Frankfurt-New York.

Boudon, R. (1998) Social Mechanisms Without Black Boxes. In: P. Hedström / R. Swedberg (Eds.) Social Mechnisms. An Analytical Approach to Social Theory, Cambridge, S. 172-203.

Bourdieu, P. / Botanski, L. / de Saint Martin, M. (1981[1971]): Kapital und Bildungskapital. Reproduktionsstrategien im sozialen Wandel, in: P. Bourdieu (Hrsg.), Titel und Stelle: über die Reproduktion sozialer Macht, Frankfurt, S. 23-87.

Broder, I. E. (1993): Professional Achievements and Gender Differences Among Academic Economists, in: Economic Inquiry XXXI, S. 116-127.

Bund-Länder-Kommission für Bildungsplanung und Forschungsförderung (2005): Frauen in Führungspositionen an Hochschulen und außerhochschulischen Forschungseinrichtungen, Heft 129, Bonn.

Burt, R. S. (1998): The Gender of Social Capital, in: Rationality and Society 10, S. 5-46.

Clark, S. M. / Corcoran, M. (1986): Perspectives on the Professional Socialization of Woman Faculty. A Case of Accumulative Disadvantage? in: The Journal of Higher Education 57, S. 20-43.

Cole, J. R. (1979) Age and Scientific Performance, in: American Journal of Sociology 89, S. 958-977.

Cole, J. R. / Cole, S. (1973): Social Stratification in Science, Chicago.

Crane, D. (1965): Scientists at Major and Minor Universities. A Study of Productivity and Recognition, in: American Sociological Review 30, S. 699-714.

Dey, E. L. / Milem, J. F. / Berger, J. B. (1997): Changing Patterns of Publication Productivity. Accumulative Advantage or Institutional Isomorphism? in: Sociology of Education 70, S. 308-323.

Enders, J. / Bornmann, L. (2001): Karriere mit Doktortitel? Ausbildung, Berufsverlauf und Berufserfolg von Promovierten, Frankfurt-New York.

Endruweit, G. (2002): Wie misst man Reputation? Messtheoretische Überlegungen zu Jürgen Gerhards »Reputation in der deutschen Soziologie«, in: Soziologie 4, S. 33-41.

Engler, S. (2001): "In Einsamkeit und Freiheit"? Zur Konstruktion der wissenschaftlichen Persönlichkeit auf dem Weg zur Professur, Konstanz.

Engler, S. (2000): Zum Selbstverständnis von Professoren und der illusio des wissenschaftlichen Feldes, in: B. Krais (Hrsg.), Wissenschaftskultur und Geschlechterordnung, Frankfurt-New York, S. 121-151.

European Commission (2006): She Figures. Women and Science. Statistics and Indicators. http://ec.europa.eu/research/science-society/pdf/she-figures-2006en.pdf, abgerufen am 20.06.07.

Feyerabend, P. (1986[1976]): Wider den Methodenzwang. Frankfurt.

Fox, M. F. (1992): Research, Teaching, and Publication Productivity. Mutuality Versus Competition in Academia, in: Sociology of Education 65, S. 293-305.

Fox, M. F. (1983): Publication Productivity among Scientists. A Critical Review, in: Social Studies of Science 13, S. 285-305.

Fox, M. F. (2005): Gender, Family Characteristics, and Publication Productivity Among Scientists, in: Social Study of Science 35, S. 131-150.

Fuchs, S. / von Stebut, J. / Allmendinger, J. (2001): Gender, Science, and Scientific Organizations in Germany, in: Minerva 39, S. 175-201. 
Gerhards, J. (2002): Reputation in der deutschen Soziologie - zwei getrennte Welten, in: Soziologie 2, S. 19-33.

Gläser, J. (2006): Die Fallstricke der Bibliometrie, in: Soziologie 35, S. 42-51.

Goodwin, T.H. / Sauer, R.D. (1995): Life Cycle Productivity in Academic Research: Evidence from Cumulative Publication Histories of Academic Economists, in: Southern Economic Journal 61, S. 728-743.

de Graaf, N. D. / Flap, H. D. (1988): "With a Little Help from My Friends". Social Resources as an Explanation of occupational Status and Income in West Germany, The Netherlands, and the United States, in: Social Forces 67, S. 452-472.

Granovetter, M. (1995[1974]): Getting a Job. A Study of Contacts and Careers, Chicago.

Granovetter, M. (1973): The Strength of Weak Ties, in: The American Journal of Sociology 78, S. 13601380.

Granovetter, M. (1983): The Strength of Weak Ties: A Network Theory Revisited, in: Sociological Theory 1, S. 201-233.

Gross, C. / Jungbauer-Gans, M. (2007): Wird man so tatsächlich Prof? Anmerkungen zum Forumsbeitrag von Plümper und Schimmelfennig, in: Politische Vierteljahresschrift 48, S. 553-558.

Gross, C. / Jungbauer-Gans, M. / Kriwy, P. (2008): Die Bedeutung meritokratischer und sozialer Kriterien für wissenschaftliche Karrieren, erscheint in: Beiträge zur Hochschulforschung.

Haller, M. (2003): Soziologische Theorie im systematisch-kritischen Vergleich, 2. Aufl., Opladen.

Hamermesh, D. S. / Parker, A. M. (2006): Beauty in the Classroom: Instructors' Pulchritude and Putative Pedagogical Productivity, in: Economics of Education Review, forthcoming.

Hartmann, I. (1988): Fachspezifische Beurteilungskriterien von Gutachtern in der Forschungsförderung - dargestellt am Beispiel des Normalverfahrens der Deutschen Forschungsgemeinschaft, in: H.-D. Daniel / R. Fisch (Hrsg.), Evaluation von Forschung, Konstanz.

Hartmann, M. (1996): Topmanager - Die Rekrutierung einer Elite, Frankfurt.

Hartmann, M. (1997): Soziale Öffnung und soziale Schließung. Die deutsche und die französische Wirtschaftselite zwischen 1970 und 1995, in: Zeitschrift für Soziologie 26, S. 296-311.

Hartmann, M. (1999): Auf dem Weg zur transnationalen Bourgeoisie? Die Internationalisierung der Wirtschaft und die Internationalität der Spitzenmanager Deutschlands, Frankreichs, Großbritanniens und der USA, in: Leviathan 27, S. 113-141.

Hartmann, M. (2001a): Bildung und andere Privilegien, in: K. M. Michel et al. (Hrsg.), Die Neidgesellschaft, Berlin, S. 39-53.

Hartmann, M. (2001b): Klassenspezifischer Habitus oder exklusive Bildungstitel als soziales Selektionskriterium? Die Besetzung von Spitzenpositionen in der Wirtschaft, in B. Krais (Hrsg.), An der Spitze. Von Eliten und herrschenden Klassen, Konstanz, S. 157-208.

Hartmann, M. (2002): Der Mythos von den Leistungseliten. Spitzenkarrieren und soziale Herkunft in Wirtschaft, Politik, Justiz und Wissenschaft, Frankfurt-New York.

Hartmann, M. / Kopp, J. (2001): Elitenselektion durch Bildung oder durch Herkunft, in: Kölner Zeitschrift für Soziologie und Sozialpsychologie 53, S. 436-466.

Hasenjürgen, B. (1996): Soziale Macht im Wissenschaftsspiel. SozialwissenschaftlerInnen und Frauenforscherinnen an der Hochschule, Münster.

Hedström, P. (2005): Dissecting the Social. On the Principles of Analytical Sociology, Cambridge.

Hedström, P. / Swedberg, R. (1998): Social Mechanisms: An Introductory Essay. In: P. Hedström / R. Swedberg (Eds.), Social Mechnisms. An Analytical Approach to Social Theory, Cambridge, S. 1-31.

Heinze, T. / Sauer, G. (2007): Characterizing Creative Scientists in Nano-S\&T: Productivity, Multidisciplinarity, and Network Brokerage in a Longitudinal Perspective, in: Scientometrics 70, S. 811-830.

Hirschauer, S. (2005): Publizierte Fachurteile. Lektüre und Bewertungspraxis im Peer Review, in: Soziale Systeme 11, S. 52-82.

Hirschauer, S. (2004): Peer Review Verfahren auf dem Prüfstand. Zum Soziologiedefizit der Wissenschaftsevaluation, in: Zeitschrift für Soziologie 33, S. 62-83. 
Hornbostel, S. (1997): Wissenschaftsindikatoren. Bewertungen in der Wissenschaft, Opladen.

Hornbostel, S. (2001): Die Hochschulen auf dem Weg in die Audit Society, in: E. Stölting und U. Schimank (Hrsg.): Die Krise der Universitäten. Leviathan Sonderheft 20, Opladen, S. 139-158.

Ibarra, H. (1992): Homophily and Differential Returns. Sex Differences in Network Structure and Access in an Advertising Firm, in: Administrative Science Quarterly 37, S. 422-447.

Joas, H. (1993): Das deutsche Universitätssystem und die Karrieremöglichkeiten junger Wissenschaftler. in: K. U. Mayer (Hrsg.), Generationsdynamik in der Forschung, Frankfurt-New York, S. 110-121.

Kermit, D. (1995): The Marriage Premium, in: M. Tommasi / K. Ierulli (Hrsg.), The New Economics of Human Behavior, Cambridge, S. 113-125.

Klein, M. / Rosar, U. (2006): Das Auge hört mit! Der Einfluss der physischen Attraktivität des Lehrpersonals auf die studentische Evaluation von Lehrveranstaltungen - eine empirische Analyse am Beispiel der Wirtschafts- und Sozialwissenschaftlichen Fakultät der Universität zu Köln, in: Zeitschrift für Soziologie 35, S. 305-316.

Krackhardt, D. (1992): The Strength of Strong Ties. The Importance of Philos in Organizations, in: N. Nohria / R.G. Eccles (Hrsg.), Boston, S. 216-239.

Krais, B. (2000a). Die Wissenschaftselite. In Michel, K. M. / Karsunke, I. / Spengler, T. (Hrsg.), Die neuen Eliten, Berlin, S. 137-146.

Krais, B. (2000b): Das soziale Feld Wissenschaft und die Geschlechterverhältnisse, in: B. Krais (Hrsg.), Wissenschaftskultur und Geschlechterordnung, Frankfurt-New York, S. 31-54.

Krais, B. (2000c): Einleitung: Die Wissenschaft und die Frauen, in: Beate Krais (Hrsg.), Wissenschaftskultur und Geschlechterordnung, Frankfurt-New York, S. 9-29.

Krimmer, H. / Stallmann, F. / Behr, M. / Zimmer, A. (2003): Karrierewege von Professorinnen an Hochschulen in Deutschland, http://www.mentoring.unizh.ch/literatur/wika_broschuere.pdf, abgerufen am 07.11.2006.

Kromrey, H. (1995): Evaluation in der Lehre durch Umfrageforschung? Methodische Fallstricke bei der Messung von Lehrqualität durch Befragung von Vorlesungsteilnehmern, in: P. P. Mohler (Hrsg.): Universität und Lehre. Ihre Evaluation als Herausforderung an die empirische Sozialforschung, MünsterNew York, S. 105-128.

Kromrey, H. (2004): Qualität und Evaluation im System Hochschule, in: R. Stockmann (Hrsg.): Evaluationsforschung. Grundlagen und ausgewählte Forschungsfelder, 2. Aufl., Opladen, S. 233-258.

Kuhlmann, E. / Matthies, H. (2001): Geschlechterasymmetrie im Wissenschaftsbetrieb, in: Berliner Journal für Soziologie 1, S. 31-50.

Kuhn, T.S. (1973[1962]): Die Struktur wissenschaftlicher Revolutionen. Frankfurt.

Lang, F.R. / Neyer, F.J. (2004): Kooperationsnetzwerke und Karrieren an deutschen Hochschulen. Der Weg zur Professur am Beispiel des Faches Psychologie, in: Kölner Zeitschrift für Soziologie und Sozialpsychologie 56, S. 520-538.

Leahey, E. (2007): Not by Productivity Alone: How Visibility and Specialization Contribute to Academic Earnings, in: American Sociological Review 72, S. 533-561

Litzenberger, T. / Sternberg, R. (2005): Die Forschungsleistung der Soziologie an zehn deutschen Universitäten. Ein bibliometrischer Vergleich auf Basis des Social Science Citation Index (SSCI), in: Soziologie 34, S. 174-190.

Litzenberger, T. / Sternberg, R. (2006): Wenn weiße Schafe krampfhaft versuchen nach schwarzen zu suchen ... und sich dabei dreckig machen! Replik auf den Beitrag von Jochen Gläser, in: Soziologie 35, S. 52-55.

Löther, A. (2006): Von der Studentin zur Professorin. Eine Analyse zum Frauen- und Männeranteil im wissenschaftlichen Qualifikationsprozess, in: Forschung \& Lehre 11, S. 634-635.

Long, S. J. (1978): Productivity and Academic Position in the Scientific Career, in: American Sociological Review 43, S. 889-908.

Long, J. S. (1990): The Origins of Sex Differences in Science, in: Social Forces 68, S. 1297-1316. 
Long, J. S. (1992): Measures of Sex Differences in Scientific Productivity, in: Social Forces 71(1), S. 159178.

Long, J. S. / Fox, M. F. (1995): Scientific Careers: Universalism and Particularism, in: Annual Review of Sociology 21, S. 45-71.

Long, J. S. / McGinnis, R. (1981): Organizational Context and Scientific Productivity, in: American Sociological Review 46, S. 422-442.

Long, J. S. / McGinnis, R. (1985): The Effects of Mentor on the Academic Career, in: Scientometrics 7, S. $255-280$.

Long, J. S. / Allison, P. D. / McGinnis, R. (1979): Entrance into the Academic Career, in: American Sociological Review 44, S. 816-830.

Maurer, A. (2006): Soziale Mechanismen und das struktur-individualistische Erklärungsprogramm. Zur forschungspraktischen Verortung sozialer Mechanismen. In: M. Schmitt / M. Florian, F. Hillebrandt (Hrsg.), Reflexive soziale Mechanismen. Von soziologischen Erklärungen zu sozionischen Modellen, Wiesbaden, S. 141-164.

Merton, R. K. (1968): The Matthew Effect in Science, in: Science 159, S.56-63.

Merton, R. K. (1985[1973]): Entwicklung und Wandel von Forschungsinteressen. Aufsätze zur Wissenschaftssoziologie, Frankfurt.

Merton, R.K. (1988): The Matthew Effect in Science, II. Cumulative Advantage and the Symbolism of Intellectual Property, in: Isis 79, S. 606-623.

Meuser, M. / Nagel, U. (2002[1991]): ExperInneninterviews - vielfach erprobt, wenig bedacht. Ein Beitrag zur qualitativen Methodendiskussion. In: A. Bogner / B. Littig / W. Menz (Hrsg.), Das Experteninterview. Theorie, Methode, Anwendung, Opladen, S. 71-93.

Merton, R.K. / Zuckerman, H. (1973): Age, Aging, and Age Structure in Science, in: R.K. Merton (Hrsg.), The Sociology of Science, Theoretical and Empirical Investigations, Chicago-London, S. 497-559.

Monks, J. / Robinson, M. (2000): Gender and Racial Earnings Differentials in Academic Labor Markets, in: Economic Inquiry 38, S. 662-671.

Mouw, T. (2003): Social Capital and Finding a job: Do contacts matter, in: American Sociological Review 68, S. 868-898.

Münch, R. (2006): Drittmittel und Publikationen, in: Soziologie 35, S. 440-461.

Neidhardt, F. (2006): Forschungsevaluationen, in: Soziologie 35, S. 419-425.

Paris, R. (2003): Autorität - Führung - Elite: Eine Abgrenzung, in: S. Hradil / P. Imbusch, Oberschichten - Eliten - Herrschende Klassen, Opladen, S. 55-72.

Pfadenhauer, M. (2002): Auf gleicher Augenhöhe reden. Das Experteninterview - ein Gespräch zwischen Experte und Quasi-Experte. In: A. Bogner / B. Littig / W. Menz (Hrsg.), Das Experteninterview. Theorie, Methode, Anwendung, Opladen, S. 113-130.

Phelps, E. S. (1972a): Inflation Policy and Unemployment Theory, New York.

Phelps, E. S. (1972b): The statistical theory of racism and sexism, in: The American Economic Review 62, S. 659-661.

Plümper, T. / Schimmelfennig, F. (2007): Wer wird Prof - und wann? Berufungsdeterminanten in der deutschen Politikwissenschaft, in: Politische Vierteljahresschrift 48, S. 97-117.

Polanyi, M. (1966): The Tacit Dimension, Garden City-New York.

Preisendörfer, P. / Voss, T. (1988): Arbeitsmarkt und soziale Netzwerke, in: Soziale Welt 39, S. 104-119.

Reskin, B. F. (1977): Scientific Productivity and the Reward Structure of Science, in: American Sociological Review 42, S. 491-504.

Rose, M. R. (1986): Pork-Barrel Science vs. Peer Review, in: Chronicle of Higher Education 08.10.1986, S. 96.

Rossiter, M. W. (1993): The Matthew Matilda Effect in Science, in: Social Studies of Science 23, S. 325-341. 
Rothböck, S. / Sacchi, S. / Buchmann, M. (1999): Die Rekrutierung der politischen, wirtschaftlichen und wissenschaftlichen Eliten in der Schweiz. Eine explorative Studie, in: Schweizerische Zeitschrift für Soziologie 25, S. 459-496.

Scandura, T. A. / Ragins B. R. (1993): The Effects of Sex and Gender Role Orientation on Mentorship in Male-Dominated Occupations, in: Journal of Vocational Behavior 43, S. 251-265.

Schimank, U. (1995): Für eine Erneuerung der institutionalistischen Wissenschaftssoziologie, in: Zeitschrift für Soziologie 24, S. 42-57.

Schluchter, W. (1996): Neubeginn durch Anpassung? Studien zum ostdeutschen Übergang, Frankfurt.

Sonnert, G. (1995): Gender Differences in Science Careers, New Brunswick.

Spence, M. (1973): Job Market Signaling, in: The Quarterly Journal of Economics 87, S. 355-374.

von Stebut, N. / Wimbauer, C. (2003): Geschlossene Gesellschaft? - Zur Integration von Frauen in der Max-Planck- und der Fraunhofer-Gesellschaft, in: H. Matthies et al. (Hrsg.), Gleichstellung in der Forschung. Organisationspraktiken und politische Strategien, Berlin, S. 105-122.

Stephan, P. / Levin, S. (1992): Striking the Mother Lode in Science. The Importance of Age, Place, and Time, Oxford.

Teichler, U. (1996): Frauen in Hochschule und Beruf - eine Sekundäranalyse der Hochschulforschung, in: S. Metz-Göckel / A. Wetterer (Hrsg.), Vorausdenken - Querdenken - Nachdenken, Frankfurt-New York, S. 89-99.

Tolbert, P. S. / Simones, T. / Alice, A. / Rhee, J. (1995): The Effect of Gender Composition in Academic Departments on Faculty Turnover, in: Industrial and Labor Relations Review 48, S. 562-579.

Weber, M. (1968): Methodologische Schriften, Studienausgabe, Frankfurt.

Wegener, B. (1991): Job Mobility and Social Ties: Social Resources, Prior Job, and Status Attainment, in: American Sociological Review 56, S. 60-71.

Weingart, P. (2003): Wissenschaftssoziologie, Bielefeld.

Wennerås, C. / Wold, A. (1997): Nepotism and sexism in peer-review, in: Nature 387, S. 341-343.

Wennerås, C. / Wold, A. (2000): Vetternwirtschaft und Sexismus im Gutachterwesen, in: B. Krais (Hrsg.), Wissenschaftskultur und Geschlechterordnung, Frankfurt-New York, S. 107-120.

Wetterer, A. (1986): Was sagen subjektive Diskriminierungserfahrungen über die objektive Situation von Wissenschaftlerinnen aus? in: B. Clemens et al. (Hrsg.), Töchter der Alma Mater, Frankfurt-New York, S. 273-286.

Wissenschaftsrat (2001): Personalstruktur und Qualifizierung: Empfehlungen zur Förderung des wissenschaftlichen Nachwuchses, Drs. 4756/01, www.wissenschaftsrat.de/texte /4756-01.pdf.

Xie, Y. / Shauman, K.A. (1998): Sex Differences in Research Productivity: New Evidence About an Old Puzzle, in: American Sociological Review 63, S. 847-870.

Zimmermann, K. (2000): Spiele mit der Macht in der Wissenschaft, Berlin.

Zuckerman, H. (1967): Nobel Laureates in Science. Patterns of Productivity, Collaboration, and Authorship, in: American Sociological Review 32, S. 391-403.

Zuckerman, H. (1968): Patterns of Name Ordering among Authors of Scientific Papers. A Study of Social Symbolism and its Ambiguity, in: American Journal of Sociology 74, S. 276-291.

Zuckerman, H. (1993): Die Werdegänge von Nobelpreisträgern, in: K. U. Mayer (Hrsg.), Generationsdynamik in der Forschung, Frankfurt-New York, S. 59-79.

Prof. Dr. Monika Jungbauer-Gans Dipl. Soz. Christiane Gross

Universität zu Kiel

Institut für Sozialwissenschaften

Westring 400

24098 Kiel

jungbauer-gans@soziologie.uni-kiel.de cgross@soziologie.uni-kiel.de 